\title{
Examination of Wide Area Control Methods to Face Inter-Area Oscillations
}

\author{
$1^{\text {st }}$ Segundo Sevilla, Felix Rafael \\ Zurich University of Applied Science \\ segu@zhaw.ch
}

$4^{\text {th }}$ Obushevs Artjoms

Zurich University of Applied Science

obus@zhaw.ch

\author{
$2^{\text {nd }}$ Dobrowolski, Jean \\ Zurich University of Applied Science \\ xdow@zhaw.ch
}

\author{
$3^{\text {rd }}$ Arrieta Paternina, Mario Roberto \\ National Autonomous University of Mexico \\ mra.paternina@fi-b.unam.mx
}

\author{
$5^{\text {th }}$ Korba Petr \\ Zurich University of Applied Science \\ korb@zhaw.ch
}

\begin{abstract}
Transmission systems are prone to experience interarea oscillations, triggered by groups of generators situated on different geographical locations oscillating against each other. In this paper, this particular problem is addressed and different control methodologies using wide-area monitoring that vary on complexity are proposed. The first approach is a proportional (P) gain that consider availability of measurements from different areas of the system and solve an optimization function to command the most influence group of machines in the system. The second approach is a Linear Quadratic Gaussian methodology that uses wide area measurements as control inputs. Finally, a proportional-integral (PI) controller that considers the availability of measurements from different areas is discussed. The first two approaches were tested through different simulations on IEEE benchmark models and the latest approach was tested using a control-in-the-loop and hardware-in-the-loop setup. To conclude, a perspective of wide-area damping controller implementation in a real laboratory demonstrator is presented.

Index Terms-Wide-Area Controller, Inter-Area Oscillation, Transmission Grid, Stability, Control-in-the-Loop, Experimentation, Particle Swarm Optimization, Linear Quadratic Gaussian.
\end{abstract}

\section{INTRODUCTION}

The continuous growth and development of society have a direct correlation to the amount of energy required to satisfy its demand [Kerber et al.(2012)]. As consequence, more interconnections of existing electrical networks are required, increasing the complexity on its operation. In some regions like Europe, this idea of super-grid is evident as demonstrated with the interconnection of Turkey in 2010 and the implementation of ambitious energy transitions across Europe [Nassar et al.(2012)]. However, more interconnection representing longer distances in a super-grid increase the risk of groups of generators reacting in a negative way, thus creating the socalled inter-area oscillations [Zhu et al.(2007)].

Moreover, with the general trend of massive penetration of renewable sources in power systems and the expected decommissioning of nuclear based energy as in Switzerland [SFOE (2017))], the general energy landscape is continuously

The authors acknowledge the Swiss National Science Foundation (SNSF) under the program Ambizione Energy Grant (AEG) with project number PZENP2_173628. changing. Despite all the obvious benefits of Renewable Energy Sources (RES), it has been recently proved [Nguyen et al (2011)], [Rueger et al (2019)] that massive integration of RES in large power grids can increase the phenomena of inter-area oscillations.

These swings are characterized for their low frequency, usually within the order of 0.1 to $1 \mathrm{~Hz}$, low damping ratios and long settling times. The result of this negative events is the limitation on the power transfer capacity and triggering of potential cascading events that might lead to blackouts [Mousavi et al(2014)], [Venkatasubramanian and Li (2004)]. Recent reported events in the European grid such as the unexpected trip of a transmission line between France and Spain [Entsoe(2017)], which caused severe oscillations of 0.1 $\mathrm{Hz}$ producing power exchanges in the order of $450 \mathrm{MW}$, motivate our interest in this particular problem.

The control of inter-area oscillations is a established area of research. However, most of the available solutions in the literature, focus on tuning Power System Stabilizer (PSS) [Dobrowolski et al.(2018)], [Stativa et al.(2012)] and design of Wide Area Damping Controllers (WADC) [Zamani et al.(2009)], [Zhang and Bose(2008)]. The main difference among the solutions lies in the architecture of the proposed controller. Two methodologies have been widely used to mitigate the problems caused by low frequency oscillations resulting from poorly damped inter-area modes [Kundur et al.(1994)], [Liu et al.(2017)], [Dobrowolski et al.(2018)]. These approaches are based on: models and measurements, respectively. In this paper, particular attention is given to WADC, since redesigning all PSS in a super-grid is unrealistic.

Designing WADC can vary in complexity from a simple proportional gain to sophisticated methods based on optimization methodologies [Zamani et al.(2009)]. In this paper, a system with $n$ number if synchronous machines is considered. The proposed WADC receives remotely measurements from Phasor Measurement Units (PMUs) and defines a new input for the excitation system, which is incorporated to the existing control of a PSS. The controller, depicted on Fig. 1, is used in a multi-area grid and three different methodologies to design the WADC are presented: 


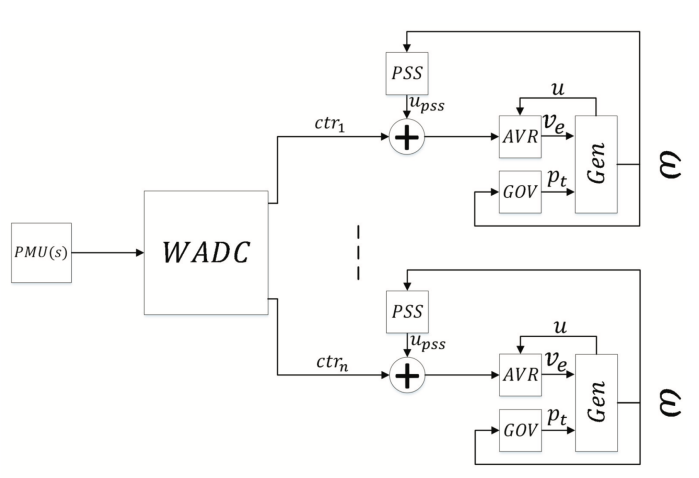

Fig. 1. Control sequence of an area with $n$ Generators (Gen) in a multiple area power systems. The Average Voltage Regulator (AVR) includes the excitation system. The Governor (GOV) is in charge of speed regulation.

1) First, a simple proportional gain that consider availability of measurements from different areas of the system and solve an optimization function using the so-called Particle Swarm Optimization (PSO) is introduced on Section II.

2) A Linear Quadratic Gaussian approach based on wide area measurements is presented on Section III.

3) Finally, a PI controller that considers the availability of measurements from different areas is described on Section IV.

Both controls (1) and (2) were tested on the IEEE-39 and the 2-areas Kundur system, respectively. Control (3) was tested on an innovative experimentation including real-time simulation, control in the loop and hardware in the loop and is presented in Section IV. Lastly, the perspective of testing WADC in a real laboratory implementation of a scaled version of the Kundur's model is discussed [Baltensperger et al (2020)].

\section{OfFline Particle SWARm Optimization to tune SIMPLE CONTROL}

\section{A. Methodology description}

Let us consider a two-areas dynamic model such as the IEEE-39 bus system, with $n$ producers. To deal with the global objective of simplification of power grid control, the first control design (1) of one area, presented on Fig. 2, is a P controller that aims to minimize the gap between frequencies of both areas. To do so, we assume that PMUs are able to measure a precise average frequency of both areas and that the communication system is fast enough for the WADC implementation. The novelty of this approach is obviously not the complexity of the controller, but the way to choose the most appropriate value for the parameters $k_{b_{i}}, i \in[1, n]$ (the P-gains).

The tuning of the parameters $k_{b_{i}}$ is solve by the PSO presented by [Dobrowolski et al (2019)]. The PSO is a computational method that optimizes a problem by iteratively trying to improve a candidate solution with regard to a given measure of quality, its convergence has been proved many

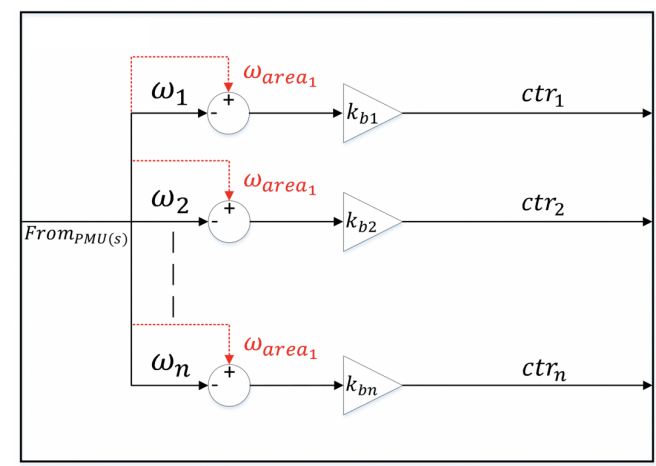

Fig. 2. Design of WADC (1) controlling area 2. PMUs are measuring frequency in both areas and the WADC objective is to minimize the gap between frequencies (speed) of both areas.

times and particularly by [Clerc and Kennedy (2002)]. In this methodology, $m$ particles represent a candidate solution for the defined optimization problem $J$, which is represented by an objective function. Every particle $i$ is characterized by:

- Position $P_{i}$ and Velocity $V_{i}$;

- A measure of quality $J\left(P_{i}\right)$;

- A best solution metric in relation to the initial state of the algorithm $P_{\text {best }_{i}}$

Moreover, considering all the particles, an additional parameter named $P_{b e s t}$ is introduced, which corresponds to global best solution such as $J\left(P_{\text {best }}\right) \leq J\left(P_{\text {best }_{i}}\right)$.

Let us consider one particle as a set of $n$ values corresponding to $k_{b_{i}}, i \in[1, n]$ (one particle $=n$ dimensions), the PSO algorithm is defined as follows:

1) First, the $m, n$ dimensional, particles are randomly placed in the searching space, restricted by limitations. An initial velocity $V_{i}$ is also defined.

2) At each iteration $k$, the position $P_{i}$ and velocity $V_{i}$ of each particles is updated according to:

- The momentum, driven by the inertia $\mathrm{H}$;

- The cognitive part, driven by the particle best solution $P_{\text {best }_{i}}$, a weighting coefficient $c_{1}$ and a random number $r$ that changes value at each iteration;

- The social part, driven by the best position of the tribune (or swarm) $P_{b e s t_{i}}$, a weighting coefficient $c_{2}$ and a random number $r$ that changes value at each iteration;

This is resumed by equation (1):

$$
\left\{\begin{aligned}
V_{i}(k+1) & =H \times V_{i}(k) \\
& +c_{1} \times r \times P_{\text {best }_{i}} \\
& +c_{2} \times r \times P_{\text {best }} \\
P_{i}(k+1) & =P_{i}(k)+V_{i}(k+1)
\end{aligned}\right.
$$

3) Step (2) is done as many times as the defined number of iterations

4) The particle that produced $P_{b e s t}$ is selected

The particularity of PSO and the quality of its results arises from the definition of the measure of quality $J\left(P_{i}\right)$. Con- 


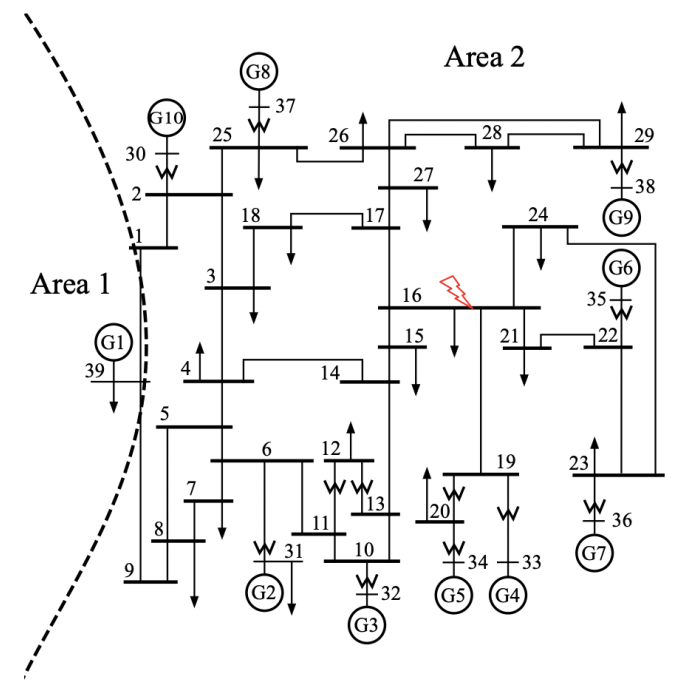

Fig. 3. IEEE-39 buses model which represent two areas and five inter-area modes.

sidering the objective of improving the damping of interarea oscillations and reducing its effect, and analyzing that the control proposed on Fig. 2 is based on generators speed measurement, the objective function $J$ in equation (2) aims to minimize the rotor speed $\left(\omega_{r}\right)$ between generators in both areas, while maintaining regulation at nominal speed $\omega_{\text {ref }}$ and minimizing the number of oscillations $N_{o s c}$.

$$
J=\sum_{i \in a_{2}}\left[\omega_{a_{1}}-\omega_{i}\right]^{2}+\sum_{i \in a_{2}}\left[\omega_{i}-\omega_{r e f}\right]^{2}+\beta N_{o s c}
$$

Where $\omega_{a_{1}}$ is the average speed in area one, $\beta$ a scaling factor and $a_{2}$ the group of generator in area two.

\section{B. Application to the IEEE-39 bus system}

Let us apply this control for the area 2 of the IEEE-39 model depicted on Fig. 3. This model has 5 inter-area modes in the range of [0.59 - 0.97] Hz. In this work, special attention is placed to the mode with the lowest frequency, $0.59 \mathrm{~Hz}$, since it is the less damped and represents the interaction between generator G1 (area 1) against the rest of the generators in area 2. Simulations lasting 5 second with $100 \mathrm{~ms}$ of sampling time and following a 3-phase short-circuit at bus 16 after 1 second of simulation were considered.

In order to perform the methodology described in the previous section, the alternation of python and the commercial software for power systems analysis DIgSILENT PowerFactory [Dobrowolski et al (2019)] were used. From python, the particles positions $P_{i}$ and Velocity $V_{i}$ are calculated, then the controls parameters $k_{b_{i}}$ are uploaded in DIgSILENT PowerFactory. RMS simulations are performed with all the particles parameters and $J$ is calculated for all the simulations. These actions are repeated as many times as the number of simulations defined. Without WADC, the objective function $J=62154.2$.

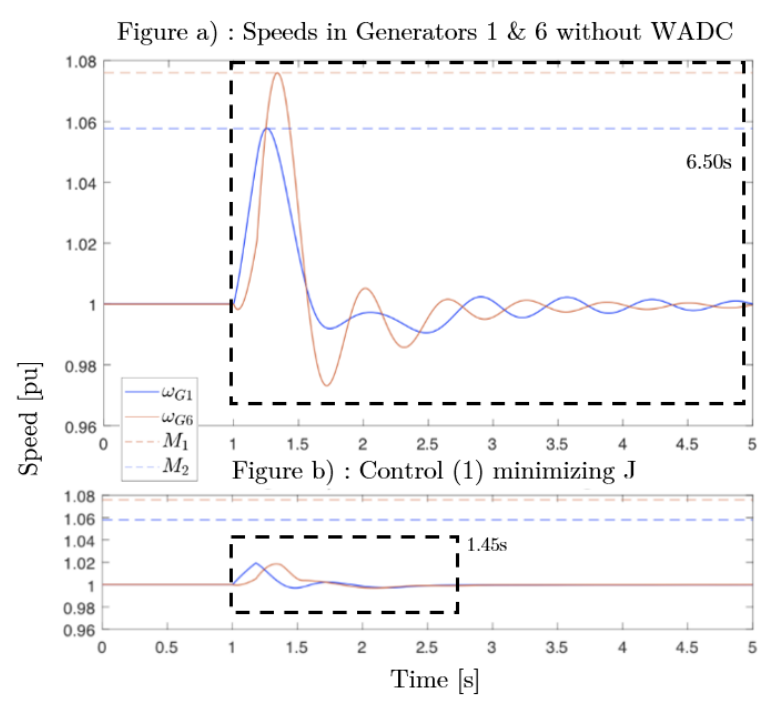

Fig. 4. Simulation result with and without WADC tuned with PSO methodology

The used parameters of equation (1) are summarize in table I. Using this configuration, optimal parameters $k_{b_{i}}$ solved by PSO are presented in table II. With this WADC configuration, the objective function $J=40533.6$ which corresponds to an improvement of around $30 \%$ of performances, according to $J$ definition.

TABLE I

PARTICLE SWARM OPTIMIZATION CONFIGURATION PARAMETERS

\begin{tabular}{|c|c|c|c|c|c|}
\hline $\begin{array}{c}\text { Inertia } \\
H\end{array}$ & $c_{1}$ & $c_{2}$ & $\begin{array}{c}\text { Number of } \\
\text { particles }\end{array}$ & $\begin{array}{c}\text { Number of } \\
\text { iteration }\end{array}$ & $\begin{array}{c}\text { Range of } \\
k_{b_{i}}\end{array}$ \\
\hline 0.3 & 0.9 & 1.1 & 100 & 70 & {$[0.01-100]$} \\
\hline
\end{tabular}

TABLE II

PARTICLE SWARM OPTIMIZATION CONFIGURATION PARAMETERS

\begin{tabular}{|c|c|}
\hline$k_{b_{1}}$ & 91.231 \\
\hline$k_{b_{2}}$ & 29.245 \\
\hline$k_{b_{3}}$ & 96.021 \\
\hline$k_{b_{4}}$ & 49.568 \\
\hline$k_{b_{5}}$ & 10.254 \\
\hline$k_{b_{6}}$ & 82.187 \\
\hline$k_{b_{7}}$ & 96.329 \\
\hline$k_{b_{8}}$ & 9.548 \\
\hline$k_{b_{9}}$ & 68.245 \\
\hline$J$ & 40533.6 \\
\hline
\end{tabular}

This improvement is highlighted on Fig.4 a) and b), respectively and it shows the simulation results with and without WADC for frequencies (speeds) of generators 1 and 6 (randomly chosen). We can clearly observe on Fig.4 b) that control (1) improves both the amplitude of the oscillations (from 1.08 p.u maximum to 1.03) and the damping (from 6.50 seconds without WADC to 1.45 seconds with). With this result, it is worth noticing that although controller (1) is a 
simple proportional gain, that was designed offline based on measurements, it allows the system to minimize the risk caused by severe events and enhance its performance. The results demonstrate that Wide Area monitoring systems can be used to considerable improve the damping of inter-area oscillations in transmission grids.

\section{ONLINE LiNEAR QuADRATIC GAUSSIAN CONTROL BASED WIDE AREA MONITORING}

\section{A. Methodology description}

Let us now consider the second WADC which is the widely used LQG controller based Wide Area Monitoring. The LQG is not a new topic and has been widely used [Dobrowolski et al.(2018)]. For this control, we consider available a state space representation of the system:

$$
\begin{cases}\dot{x} & =A_{r} x+B_{r} u \\ y & =C_{r} x\end{cases}
$$

Since the control sequence of the LQG (the so-called Linear Quadratic Regulator) is $u=-K_{r} x$ with $K$ the solution of the quadratic minimization problem, it requires the knowledge of state vector $x$ which, in the case of power systems, corresponds to voltage magnitudes and angle as well as generator parameters that cannot be accurately retrieved. Thus, the association of a Linear Quadratic Estimator (LQE) is needed in order to form the LQG controller. This LQE is based on a Kalman filter $G$ which allows an estimation $\hat{x}$ of $x$ following the equation:

$$
\left\{\begin{array}{l}
\dot{\hat{x}}=A_{r} \hat{x}+B_{r} u+G_{r}(y-\hat{y}) \\
\hat{y}=C_{r} \hat{x}
\end{array}\right.
$$

In this form, from the model matrices $A_{r}, B_{r}$ and $C_{r}$, the LQG parameters $K_{r}$ and $G_{r}$ can be calculated as in [Dobrowolski et al.(2018)].

\section{B. Application to the 11-buses Kundur system}

The system depicted on Fig. 5 will be considered in this section. Since the LQG controller aims to deal with inter-area oscillations, the identified model (3) consider $y \in \mathbb{R}^{4}$ as the variations between the rotor speeds of the two generators in area 2 with the average speed of area 1 and inversely, and $u \in \mathbb{R}^{4}$ as the PSS output. Then, a 15 order model is identified using a subroutine such as Matlab/N4SID.

Once the state space representation (3) is known, the LQG gains $K_{r}$ and $G_{r}$ can be computed and a RMS simulation of the Kundur system, following a three-phase short-circuit at bus labeled as 101 at time $t=1.0$ s is performed. Fig. 6 shows the active power in the four tie-lines in the middle of the model, without WADC (Fig.6 a) and with WADC (Fig.6 b). Without controller, Fig. 6 a shows large exchanges of active power due to inter-area oscillations between area 1 and 2 . This exchange is damped after 19.02s by existing PSS, AVR and GOV. After incorporation of the WADC on Fig. 6 b, it can be observed how the active power flow is stabilized after $7.45 \mathrm{~s}$ and about 3 oscillations. The proposed WADC, applied on a simpler model, shows how the damping of inter-area oscillations can be improved up to $60 \%$ more.

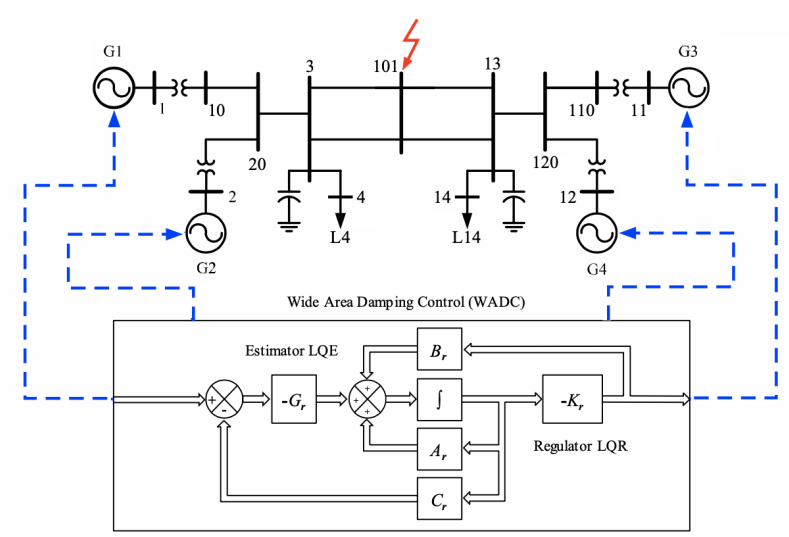

Fig. 5. Application of LQG methodology on the Kundur 11-buses model.

Figure a) : Tie-line active power without WADC

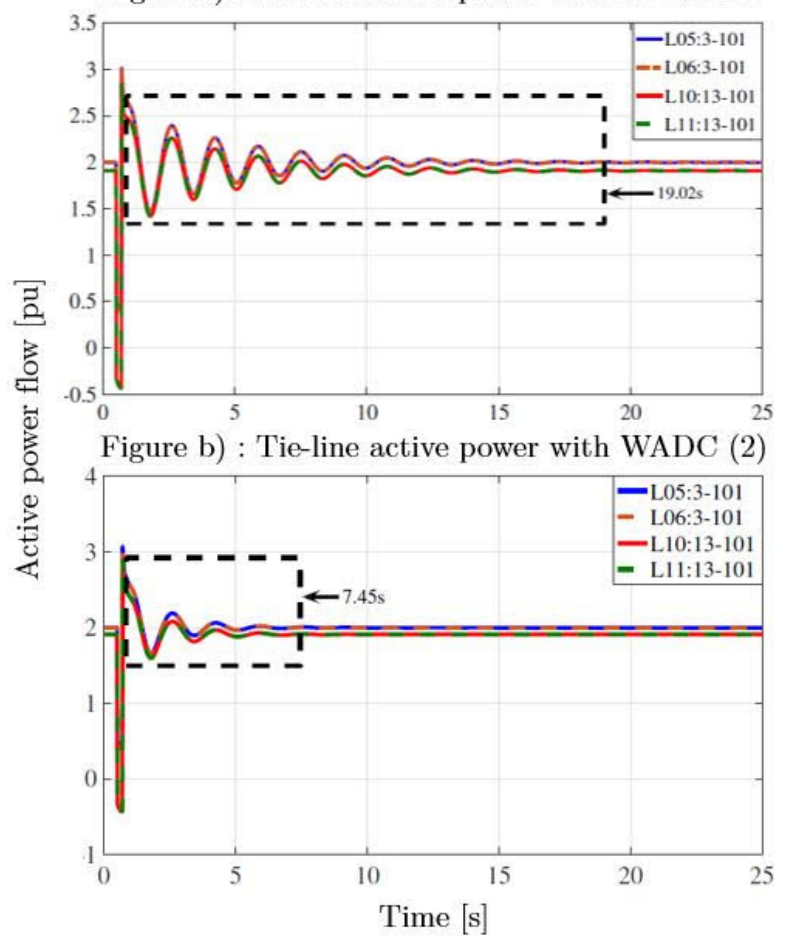

Fig. 6. Application of LQG methodology on the Kundur 11-buses model.

\section{WADC IN REAL-TIME SIMULATION COUPLED WITH CONTROL-IN-THE-LOOP}

In this section, the novelty does not lie in the structure of the controller but in the innovative of the implementation. Indeed, all the controls presented in this document concern to transmission systems, which cannot (or hardly) be tested on the field. Similarly to power system controllers, there are other fields, where the behavior of the components cannot be accurately modeled and therefore software simulation tests can be insufficient to validate the proper functionality of the systems. 


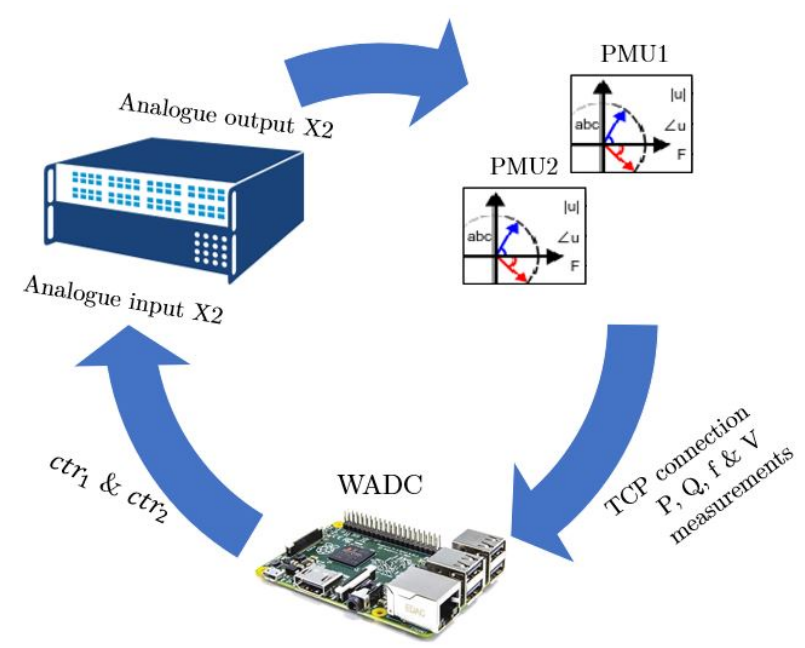

Fig. 7. Real-time simulation added to Hardware \& Control-in-the-loop setup.

Particularly in control area, an over estimated gain can excite unknown high-gain term in a traditional equation [Dobrowolski et al (2017)]. To confront these demonstration challenges, some others solutions exists to validate the effectiveness of a WADC, for example:

- Real-time simulation in order to challenge computing time of the controller [Bhadu and Senroy (2017)]

- Hardware-in-the-loop to integrate measurement errors in the testing [Li et al (2016)]

- Consideration of time delays [He et al (2009)]

Since the proposed WADC should be integrated on a PLC or a microcontroller device, two main challenges arise: measurement noise/error and control noise during the communication. In order to integrate both uncertainties in the demonstration, the following setup is proposed and depicted on Fig. 7:

- The Matlab Kundur system proposed by [Mathworks (2009)] is simulated in Opal-RT real-time simulator

- Analogue output of Opal-RT simulator are connected to Phasor Measurement Units (PMUs) from National Instruments

- Analogue input of Opal-RT simulator are connected to Raspberry Pi board which implement the WADC in Python. Since Raspberry Pi does not include analogue output, the additional board ADS1256 DAC8552 AD / DA with two analogue outputs is included.

Since the overall objective of the proposed control is to damp inter-area oscillation and reduce its impact on the power exchanges, the objective of the controller is to reduce the gap between the frequencies of area 1 and 2 but also to control their reference value $f_{\text {ref }}$. Thus, the error $e_{i}$ with $i$ representing the area of interest, is introduced as follows:

$$
e_{i}=\left(f_{\text {area }_{j}}-f_{\text {area }_{i}}\right)+\left(f_{\text {ref }}-f_{\text {area }_{i}}\right)
$$

where $j$ is the second area. Then, the control of area $i$ is a single PI controller which aims to minimize $e_{i}$ :

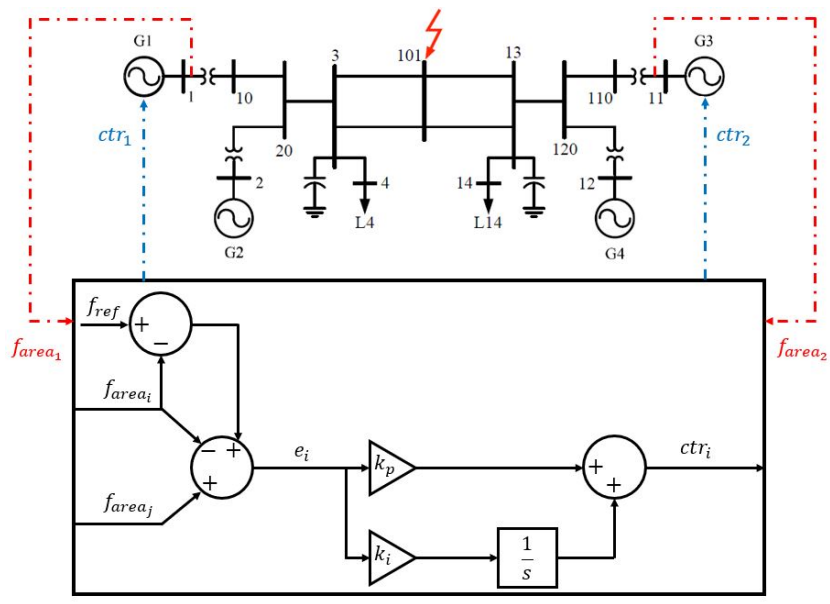

Fig. 8. Summary of connections between the real time simulated model and the control implemented in the raspberry Pi board.

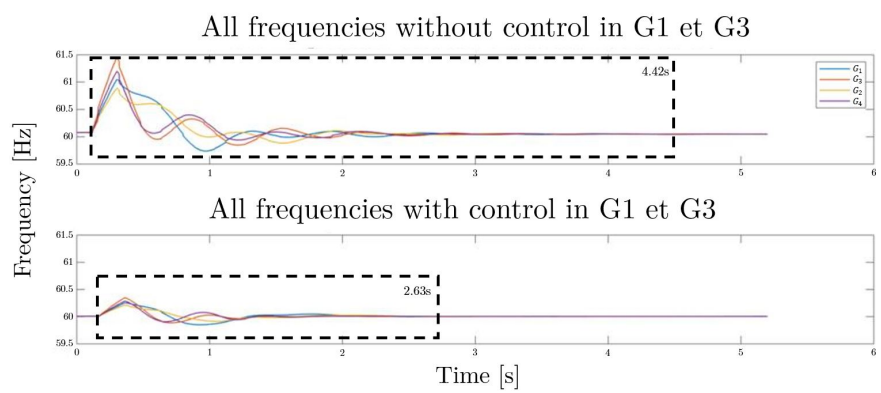

Fig. 9. Frequencies of the four generators without (top) and with (bottom) WADC connected to generators $1 \& 3$.

$$
c t r_{i}=k_{p} e_{i}+k_{i} \frac{e_{i}}{s}
$$

where $k_{p}$ and $k_{i}$ are the gains of the controller. Thus, considering the setup depicted on Fig. 7, the controller defined on Eq.(6) is applied to generators 1 and 3, respectively. Frequencies of both areas are measured at buses 1 and 11 following a 3-phase short circuit at bus 101 at $t=0.1 \mathrm{~s}$. The connections between the model and the controller are summarized on Fig. 8.

Finally, Fig. 9 shows the frequencies of the four generators of the model without (top of figure 9) and with (bottom of figure 9) WADC connected to generators $1 \& 3$.

From the results, it can be observed that without control, the maximum deviation is $1.5 \mathrm{~Hz}$ and generators are oscillating during $4.42 \mathrm{~s}$, showing an important disturbance in the grid. After, adding the WADC, the maximum deviation is around $0.4 \mathrm{~Hz}$ and the oscillations are damped after 2.63s. It is important to note that the additional board in the Raspberry pi introduced new precision and noise constraints. Therefore an additional filter and a ramp limiter were integrated in order to avoid fast changes. The results show that using a simple WADC, inter-area oscillations can be significantly improved also in a real demonstrator. 


\section{CONCLUSION}

In this paper, three different ways to design effective Wide Area Damping Controllers (WADC) have been presented. The methodologies vary in complexity from a simple proportional gain to a sophisticated iterative approach. Simulation results in two different IEEE benchmark models demonstrate the effectiveness of the proposed methodologies. The simulations compare the results of the proposed schemes against traditional solutions. The innovative part of the proposed controllers is its implementation rather than the methodology itself. Since control design of transmission systems is a conservative domain where is not possible to test state-of-the-art algorithms on field, in the last section a simple approach has been demonstrated using a real lab setup based on real-time simulators, microcontrollers (Raspberry-pi) and real communication systems (PMUs). The results validate that the proposed controllers based on remote signals can effectively be used to damp interarea oscillations, even in the presence of additional constraints arising from the communication infrastructure such as noise and measurement errors.

\section{REFERENCES}

[Kerber et al.(2012)] Kerber, G., Witzmann, R., \& Sappl, H. (2009, May) Voltage limitation by autonomous reactive power control of grid connected photovoltaic inverters. In 2009 Compatibility and Power Electronics (pp. 129-133). IEEE.

[Nassar et al.(2012)] Nassar, I. A., \& Weber, H. (2012). System analysis of the Turkish power system for interconnection with continental Europe. IFAC Proceedings.

[SFOE (2017))] Energy Strategy 2050, Swiss Federal Office of Energy (SFOE), 2017. [Online]. Available: http://www.bfe.admin.ch/energiestrategie2050/ index.html?lang=en

[Zhu et al.(2007)] F. Zhu, H.-G. Zhao, Z.-H. Liu, and H.-Z. Kou, The influence of large power grid interconnected on power system dynamic stability, Proceedings of the CSEE, 2007.

[Mousavi et al(2014)] Mousavi, O. A., Bozorg, M., Cherkaoui, R., \& Paolone, M. (2014). Inter-area frequency control reserve assessment regarding dynamics of cascading outages and blackouts. Electric power systems research.

[Venkatasubramanian and $\mathrm{Li}(2004)]$ V. Venkatasubramanian and $\mathrm{Y} . \mathrm{Li}$, Analysis of 1996 western american electric blackouts, Bulk Power System Dynamics and Control-VI, Cortina d-Ampezzo, 2004.

[Entsoe(2017)] Analysis of ce inter-area oscillations of 1st december 2016, entsoe, 2016, available at https://docstore.entsoe.eu/Documents/SOC20 documents/Regional GroupsContinentalEurope/2017/ CEinterareaoscillationsDec1st2016PUBLICV7.pdf.

[Dobrowolski et al.(2018)] Dobrowolski, J., Segundo, F. R., \& Paternina, M. R. A. (2018, November). Inter-area oscillation control based on eigensystem realization approach. In 2018 IEEE International Autumn Meeting on Power, Electronics and Computing (ROPEC).

[Stativa et al.(2012)] A. Stativa, M. Gavrilas, and V. Stahie, Optimal tuning and placement of power system stabilizer using particle swarm optimization algorithm, in Electrical and Power Engineering (EPE), 2012.

[Zamani et al.(2009)] M. Zamani, N. Sadati, and M. K. Ghartemani, Design of an $\mathrm{h}$ pid controller using particle swarm optimization, International Journal of Control, Automation and Systems, 2009.

[Zhang and Bose(2008)] Y. Zhang and A. Bose, Design of wide-area damping controllers for interarea oscillations, IEEE Transactions on Power Systems, Aug 2008.

[Kundur et al.(1994)] P. Kundur, N. J. Balu, and M. G. Lauby, Power system stability and control. McGraw-hill New York, 1994.

[Liu et al.(2017)] H. Liu, L. Zhu, Z. Pan, F. Bai, Y. Liu, Y. Liu, M. Patel, E. Farantatos, and N. Bhatt, Armax-based transfer function model identification using wide-area measurement for adaptive and coordinated damping control, IEEE Transactions on Smart Grid.
[Nguyen et al (2011)] Nguyen, M. H., Saha, T. K., \& Eghbal, M. (2011, September). Impact of high level of renewable energy penetration on inter-area oscillation. In AUPEC 2011.

[Rueger et al (2019)] Rueger, C., Dobrowolski, J., Korba, P., \& Sevilla, F. R. S. (2019, September). Lyapunov exponent for evaluation and ranking of the severity of grid events on extra-large power systems. In 2019 IEEE PES Innovative Smart Grid Technologies Europe (ISGT-Europe).

[Baltensperger et al (2020)] Baltensperger, D., Dobrowolski, J., Obushevs, A., Sevilla, F. R. S., \& Korba, P. (2020, June). Scaling Version of Kundur's Two-Areas System for Electromechanical Oscillations Representation. In 2020 International Symposium on Power Electronics, Electrical Drives, Automation and Motion (SPEEDAM).

[Dobrowolski et al (2019)] Dobrowolski, J., Korba, P., Sevilla, F. R. S., \& Sattingert, W. (2019, June). Centralized wide area damping controller for power system oscillation problems. In 2019 IEEE Milan PowerTech.

[Clerc and Kennedy (2002)] M. Clerc and J. Kennedy, The particle swarmexplosion, stability, and convergence in a multidimensional complex space, IEEE transactions on Evolutionary Computation, 2002.

[Dobrowolski et al (2017)] Dobrowolski, J., Alamir, M., Bacha, S., Gualino, D., \& Wang, M. X. (2017). On higher order dynamics in the fundamental equation of frequency in islanded microgrids. IFAC-PapersOnLine.

[Bhadu and Senroy (2017)] Bhadu, M., \& Senroy, N. (2014, October). Real time simulation of a robust LQG based wide area damping controller in power system. In IEEE PES Innovative Smart Grid Technologies, Europe.

[Li et al (2016)] Li, Y., Zhou, Y., Liu, F., Cao, Y., \& Rehtanz, C. (2016). Design and implementation of delay-dependent wide-area damping control for stability enhancement of power systems. IEEE Transactions on Smart Grid.

[He et al (2009)] He, J., Lu, C., Wu, X., Li, P., \& Wu, J. (2009). Design and experiment of wide area HVDC supplementary damping controller considering time delay in China southern power grid. IET generation, transmission \& distribution.

[Mathworks (2009)] Mathworks, PMU (PLL based, Positive Sequence) Kundur's Two-Area System. [Online]. Available: https://fr.mathworks.com/help/physmod/sps/ug/pmu-pll-based-positivesequence-kundur-s-two-area-system.html 